\title{
Doubly blind spots in scalar dark matter models
}

\author{
Wolfgang Altmannshofer, ${ }^{*}$ Brian Maddock, ${ }^{\dagger}$ and Stefano Profumo \\ Department of Physics and Santa Cruz Institute for Particle Physics, University of California, \\ Santa Cruz, California 95064, USA
}

(Received 8 July 2019; published 23 September 2019)

\begin{abstract}
We consider a framework where the Standard Model is augmented by a second $S U(2)$ scalar doublet and by a real scalar singlet that, protected by a $Z_{2}$ symmetry, provides a particle dark matter candidate. We show that this setup allows for doubly blind spots at both collider searches for anomalies in the Higgs invisible decay width, and at direct dark matter detection. The blind spots originate from cancellations between interfering diagrams featuring different neutral scalar exchanges and from cancellations driven by the twoHiggs doublet structure in the vertex coupling the singlet state with the Standard-Model-like Higgs. We demonstrate that the blind spots arise in a wide and generic array of realizations for the two-Higgs doublet model, including scenarios with a nontrivial flavor structure. We provide analytical formulas that describe the location of the blind spots in the theory parameter space, and we discuss the resulting phenomenology.
\end{abstract}

DOI: 10.1103/PhysRevD.100.055033

\section{INTRODUCTION}

The physical origin and nature of the dark matter (DM) that permeates the universe and underpins the formation and evolution of structure is at present unknown (see e.g., [1,2] for a review). A compelling possibility is that of weakly interacting massive particles (WIMPs) [3], new massive particles, neutral or close-to-neutral under electromagnetic and strong interactions, but possibly charged, or mixed with particles which are charged under weak interactions. Perhaps the most minimal possibility of the former is a new, very massive $S U(2)$ multiplet (a possibility known as "minimal DM" [4]), and of the latter is a new real scalar singlet $(S)$ that interacts via a quartic coupling with the standard model (SM) Higgs [5-11]. Here, we will be concerned with this second possibility.

Part of the appeal of WIMPs is that they are, generically, in thermal equilibrium in the early universe, eventually decoupling (freezing out) with an abundance that often is in the correct range to explain the observed amount of DM in the universe. While a far-ranging program of direct and indirect searches for WIMPs has been under way for decades now, no conclusive signal has yet been reported [12-16]: strong constraints exist as a result. In particular,

\footnotetext{
*waltmann@ucsc.edu

†bmaddock@ucsc.edu

*sprofumo@ucsc.edu
}

Published by the American Physical Society under the terms of the Creative Commons Attribution 4.0 International license. Further distribution of this work must maintain attribution to the author(s) and the published article's title, journal citation, and DOI. Funded by SCOAP. the simple possibility of a singlet scalar mentioned above $(\mathrm{SM}+S)$ is very strongly constrained; see e.g., [17].

Here, we consider a slight extension of the singlet scalar DM model to a framework where the SM is enriched with a second Higgs doublet (two-Higgs doublet model, or 2HDM) in addition to the real scalar singlet (we thus dub this scenario $2 \mathrm{HDM}+S$ ). This case's phenomenology is significantly richer, ultimately because multiple new particles now couple to the DM candidate. In particular, here we are concerned with the possibility that blind spots arise in the $2 \mathrm{HDM}+S$ as a result of either (i) destructive interference between diagrams involving different neutral scalars or (ii) exact cancellations in the $S$ coupling to the SM-like Higgs.

Blind spots have been pointed out in the literature before; see e.g., [18-29] in the context of supersymmetry and $[30,31]$ in the context of a two-Higgs doublet model. However, the central point we make here is that the blind spots in the $2 \mathrm{HDM}+S$ scenario are largely generic; i.e., they arise in a wide variety of realizations for the implementation of the specific 2HDM. Second, we point out that blind spots pertain to both collider searches (where one looks for a deviation of the invisible Higgs decay width from standard expectations) and direct DM detection, and that, on occasion, the two blind spots can overlap.

The outline of our study is as follows: In Sec. II we lay out the basic ingredients and parameters of the $2 \mathrm{HDM}+S$ model that we will discuss. In Sec. III we give an in-depth look at how to form blind spots in a generic $2 \mathrm{HDM}+S$ setup. In Sec. IV we consider the broader implication of these blind spots and how they can open up parameter space that has previously been ruled out. Finally, we conclude in Sec. V. 


\section{GENERIC TWO HIGGS DOUBLET MODEL + SINGLET}

We consider a $2 \mathrm{HDM}$ with a generic flavor structure, augmented with a real scalar singlet charged under a $Z_{2}$ discrete symmetry: This means that we extend the SM with a second Higgs doublet that can also couple to the SM fermions, as well as a scalar singlet. We consider the most generic Lagrangian for a 2HDM [32]. The part of the Lagrangian that describes the Yukawa couplings of the two Higgs doublets, $\phi_{1}$ and $\phi_{2}$, with the SM fermions in a generic 2HDM looks like

$$
\begin{aligned}
-\mathcal{L}_{\mathrm{Yuk}}= & \sum_{i, j}\left(\lambda_{i j}^{u}\left(\bar{Q}_{i} u_{j}\right) \tilde{\phi}_{1}+\lambda_{i j}^{d}\left(\bar{Q}_{i} d_{j}\right) \phi_{1}+\lambda_{i j}^{e}\left(\bar{\ell}_{i} e_{j}\right) \phi_{1}\right. \\
& \left.+\lambda_{i j}^{\prime u}\left(\bar{Q}_{i} u_{j}\right) \tilde{\phi}_{2}+\lambda_{i j}^{\prime d}\left(\bar{Q}_{i} d_{j}\right) \phi_{2}+\lambda_{i j}^{\prime e}\left(\bar{\ell}_{i} e_{j}\right) \phi_{2}\right) \\
& + \text { H.c. }
\end{aligned}
$$

for $\phi_{1}$ the "SM-like" SU(2) doublet and $\phi_{2}$ the additional doublet, and $\tilde{\phi}_{i}=i \sigma_{2} \phi_{i}^{*}$. After electroweak symmetry breaking, assuming no $C P$ violation, the $2 \mathrm{HDM}$ results in five physical Higgs bosons: a light neutral scalar $h$ (which we identify with the $125 \mathrm{GeV}$ Higgs), a heavy neutral scalar $H$ (heavy Higgs), a pseudoscalar $A$, and two charged Higgs bosons $H^{ \pm}$.

The flavor structures in 2HDMs are determined by the choice of Yukawa matrices $\lambda^{u}, \lambda^{\prime u}$, and $\lambda^{d}$, $\lambda^{\prime d}$, which then fix the couplings of the fermions to the various Higgs bosons. Common choices of these Yukawa matrices lead to four well studied models with "natural flavor conservation": type 1A, type 2A, flipped A, and lepton-specific A. The common aspect of these models is that they avoid flavor-changing neutral currents at tree level. This is achieved by coupling all three generations of one type of fermion to the same Higgs doublet [33]. The four ways that this can be done are shown in Table I.

TABLE I. Summary of the way in which the SM quarks and leptons couple to the Higgs doublets $\Phi$ and $\Phi^{\prime}$ in each of the considered models. In the models with natural flavor conservation (A), all three generations of each fermion type couple to the same Higgs doublet. In the flavorful models (B), the first two generations and the third generation couple to different Higgs doublets.

\begin{tabular}{lcccccc}
\hline \hline Model & $u, c$ & $t$ & $d, s$ & $b$ & $e, \mu$ & $\tau$ \\
\hline Type 1A & $\phi_{1}$ & $\phi_{1}$ & $\phi_{1}$ & $\phi_{1}$ & $\phi_{1}$ & $\phi_{1}$ \\
Type 1B & $\phi_{2}$ & $\phi_{1}$ & $\phi_{2}$ & $\phi_{1}$ & $\phi_{2}$ & $\phi_{1}$ \\
Type 2A & $\phi_{1}$ & $\phi_{1}$ & $\phi_{2}$ & $\phi_{2}$ & $\phi_{2}$ & $\phi_{2}$ \\
Type 2B & $\phi_{2}$ & $\phi_{1}$ & $\phi_{1}$ & $\phi_{2}$ & $\phi_{1}$ & $\phi_{2}$ \\
Flipped A & $\phi_{1}$ & $\phi_{1}$ & $\phi_{2}$ & $\phi_{2}$ & $\phi_{1}$ & $\phi_{1}$ \\
Flipped B & $\phi_{2}$ & $\phi_{1}$ & $\phi_{1}$ & $\phi_{2}$ & $\phi_{2}$ & $\phi_{1}$ \\
Lepton-specific A & $\phi_{1}$ & $\phi_{1}$ & $\phi_{1}$ & $\phi_{1}$ & $\phi_{2}$ & $\phi_{2}$ \\
Lepton-specific B & $\phi_{2}$ & $\phi_{1}$ & $\phi_{2}$ & $\phi_{1}$ & $\phi_{1}$ & $\phi_{2}$ \\
\hline \hline
\end{tabular}

One can also construct models that allow for flavorchanging neutral currents while being experimentally consistent, such as flavorful 2HDMs (F2HDM) [34-37] (for related models see e.g., [38-41]). F2HDMs differ from the models with natural flavor conservation in that they treat the third generation independently from the first two generations; this means that the third generation fermions couple dominantly to the opposite Higgs doublet than their first and second generation counterparts. There are four F2HDMs, mirroring the four flavor diagonal models, referred to as type 1B, type $2 \mathrm{~B}$, flipped B, and lepton-specific B. For an indepth discussion see [36]. The flavor structures of the four flavorful models are summarized in Table I.

As we will see, the flavor structure of the quarks has the largest impact on the phenomenology of the DM in these models. Of the eight possibilities discussed above (four "type A" models and four "type B" models) there are only four different ways to couple the quarks. Both up- and down-type quarks can be coupled in the same way (type $1 \mathrm{~A} / \mathrm{B}$, lepton-specific $\mathrm{A} / \mathrm{B}$ ) or they can be coupled in the opposite way (type 2A/B, flipped A/B). Therefore, without loss of generality, for this analysis we will focus on the type $1 \mathrm{~A} / \mathrm{B}$ and type $2 \mathrm{~A} / \mathrm{B}$ models as they represent the four unique ways to couple the quarks.

The characteristic pattern of Higgs couplings to the SM quarks in the different types of $2 \mathrm{HDMs}$ is determined by two angles: $\alpha$ and $\beta$, where $\alpha$ is the mixing between the two neutral scalar components of the doublets $\phi_{1}$ and $\phi_{2}$, and $\tan \beta=v_{1} / v_{2}$ is the ratio of the vacuum expectation values of $\phi_{1}$ and $\phi_{2}$. The corresponding terms in the Lagrangian which contain the physical scalar Higgs bosons and quarks can be written as

$$
\mathcal{L} \supset \sum_{q} \bar{q} q\left(y_{q, h} h+y_{q, H} H\right),
$$

where $y_{q, h}$ and $y_{q, H}$ represent the flavor diagonal couplings of the quarks $q$ to the SM-like and heavy Higgs, respectively. As discussed, these couplings are characteristic for a given type of 2HDM. Concretely, in our four example scenarios, the couplings of the SM-like Higgs can be expressed in terms of $\alpha$ and $\beta$ as

$$
\begin{aligned}
& y_{t, h}=\frac{m_{t}}{v} \frac{\cos \alpha}{\sin \beta} \quad \text { all types, } \\
& y_{b, h}=\frac{m_{b}}{v} \times\left\{\begin{array}{ll}
\frac{\cos \alpha}{\sin \beta} & \text { type 1A, 1B } \\
-\frac{\sin \alpha}{\cos \beta} & \text { type 2A, 2B }
\end{array}\right. \text {, } \\
& y_{c(u), h}=\frac{m_{c(u)}}{v} \times\left\{\begin{array}{ll}
\frac{\cos \alpha}{\sin \beta} & \text { type 1A, 2A } \\
-\frac{\sin \alpha}{\cos \beta} & \text { type 1B, 2B }
\end{array}\right. \text {, } \\
& y_{s(d), h}=\frac{m_{s(d)}}{v} \times\left\{\begin{array}{ll}
\frac{\cos \alpha}{\sin \beta} & \text { type 1A, 2B } \\
-\frac{\sin \alpha}{\cos \beta} & \text { type 1B, 2A }
\end{array}\right. \text {, }
\end{aligned}
$$


where $v=\sqrt{v_{1}^{2}+v_{2}^{2}} \simeq 246 \mathrm{GeV}$ is the SM Higgs vacuum expectation value (VEV). For the heavy Higgs boson we have

$$
\begin{gathered}
y_{t, H}=\frac{m_{t}}{v} \frac{1}{\tan \beta} \frac{\sin \alpha}{\cos \beta} \quad \text { all types, } \\
y_{b, H}=\frac{m_{b}}{v} \times \begin{cases}\frac{1}{\tan \beta} \frac{\sin \alpha}{\cos \beta} & \text { type 1A, 1B } \\
\tan \beta \frac{\cos \alpha}{\sin \beta} & \text { type 2A, 2B }\end{cases} \\
y_{c(u), H}=\frac{m_{c(u)}}{v} \times\left\{\begin{array}{ll}
\frac{1}{\tan \beta} \frac{\sin \alpha}{\cos \beta} & \text { type 1A, 2A } \\
\tan \beta \frac{\cos \alpha}{\sin \beta} & \text { type 1B, 2B }
\end{array},\right. \\
y_{s(d), H}=\frac{m_{s(d)}}{v} \times\left\{\begin{array}{ll}
\frac{1}{\tan \beta} \frac{\sin \alpha}{\cos \beta} & \text { type 1A, 2B } \\
\tan \beta \frac{\cos \alpha}{\sin \beta} & \text { type 1B, 2A }
\end{array} .\right.
\end{gathered}
$$

Additional small corrections to the couplings are present in the flavorful models (type 1B and type 2B). They are proportional to small ratios of fermion masses and can be found in [36].

The other ingredient in the framework we consider here is a real scalar singlet $S$, which is assumed to be charged under a discrete $Z_{2}$ symmetry. This scalar singlet only interacts with the SM through the "Higgs portal," i.e., through gauge-invariant, renormalizable operators of the type $S^{2} \phi_{i}^{\dagger} \phi_{j}$. The terms in the scalar potential that contain the singlet $S$ are

$$
\begin{aligned}
\mathcal{V}_{S}= & m_{S}^{2} S^{2}+\lambda_{S} S^{4}+\lambda_{S_{1}}\left|\phi_{1}\right|^{2} S^{2}+\lambda_{S_{2}}\left|\phi_{2}\right|^{2} S^{2} \\
& +\left(\lambda_{S_{12}} \phi_{1}^{\dagger} \phi_{2}+\lambda_{S_{12}}^{*} \phi_{2}^{\dagger} \phi_{1}\right) S^{2} .
\end{aligned}
$$

We assume that $m_{S}^{2}$ is positive such that $S$ does not obtain a vacuum expectation value and the $Z_{2}$ symmetry that stabilizes $S$ remains unbroken. The quartic interactions between the singlet $S$ and the doublets $\phi_{1}$ and $\phi_{2}$ are parametrized by the real couplings $\lambda_{S_{1}}$ and $\lambda_{S_{2}}$ and the in general complex coupling $\lambda_{S_{12}}$.

In order to obtain the couplings of the DM $S$ to the physical Higgs bosons the Lagrangian must be rotated to the mass basis. Defining the interactions with the mass eigenstates as

$$
\mathcal{L} \supset S^{2}\left(h g_{S S h}+H g_{S S H}+A g_{S S A}\right),
$$

we find

$$
\begin{aligned}
g_{S S h}= & v\left(\lambda_{S_{1}} \sin \beta \cos \alpha-\lambda_{S_{2}} \cos \beta \sin \alpha+\operatorname{Re}\left(\lambda_{S_{12}}\right)\right. \\
& \times(\cos \beta \cos \alpha-\sin \beta \sin \alpha)), \\
g_{S S H}= & v\left(\lambda_{S_{1}} \sin \beta \sin \alpha+\lambda_{S_{2}} \cos \beta \cos \alpha\right. \\
& \left.+\operatorname{Re}\left(\lambda_{S_{12}}\right)(\sin \beta \cos \alpha+\cos \beta \sin \alpha)\right), \\
g_{S S A}= & -v \Im\left(\lambda_{S_{12}}\right) .
\end{aligned}
$$

In the following we will assume that the tree-level scalar potential conserves $C P$, and therefore set $\Im\left(\lambda_{S_{12}}\right)=0$, such that there are no couplings between the dark matter and a single pseudoscalar Higgs. This choice has little impact on our main results. The pseudoscalar interactions lead to spin dependent dark matter scattering, and the corresponding bounds are several orders of magnitude weaker than those from spin independent scattering mediated by the scalars.

\section{EXPERIMENTAL CONSTRAINTS AND BLIND SPOTS}

We consider four constraints on the framework under consideration: the thermal relic density of the dark matter candidate (which we enforce to be reflective of the universal dark matter density), spin-independent direct detection, indirect detection via gamma-ray observations, and invisible Higgs decays. Other DM models with extended Higgs sectors can also be constrained by di-Higgs + missing transverse energy (MET) searches, but these constraints are weak in this model [42]. In this section we show how the parameters of this model can conspire such to create blind spots in the constraints from direct detection experiments and invisible Higgs decays.

The relic density refers to the abundance of DM particles left over from freeze-out in the early universe versus the inferred abundance of cosmological DM. The latter was measured by PLANCK (utilizing other data sets as well) to be $\Omega h^{2}=0.1198 \pm 0.0015$ [43]. Any viable DM candidate must predict the relic density to be no greater than $\Omega h^{2}$, barring modification to the universe's expansion history. In our model we consider a standard freeze-out scenario where the DM is in thermal equilibrium with the SM in the early universe, which we assume to be radiation dominated. At this time the DM can annihilate into SM particles, but eventually falls out of thermal equilibrium leaving behind some relic abundance.

DM is abundant in many astrophysical objects, and the annihilation of DM into SM particles can generically lead to an excess of gamma rays. Indirect detection searches for signatures of DM in gamma ray spectra, and sets constraints on DM models in the absence of any significant excess over background [12-15]. Notice that the annihilation of DM into SM particles is a relevant process for both determining the relic abundance and understanding indirect detection, so these two processes are correlated, even though the relevant center-of-mass energy for the thermal decoupling process is biased at slightly larger values since the decoupling happens at finite temperature.

Direct detection experiments use nucleons as a target for DM to scatter. When the DM scatters off of nuclei, the latter subsequently recoil; this recoil can then be measured and provides information on the mass and coupling of the DM (see e.g., [44] for a recent review). In simple scalar DM models the Higgs mediates the DM-nucleon interaction via direct interaction with the light constituent quarks of the 


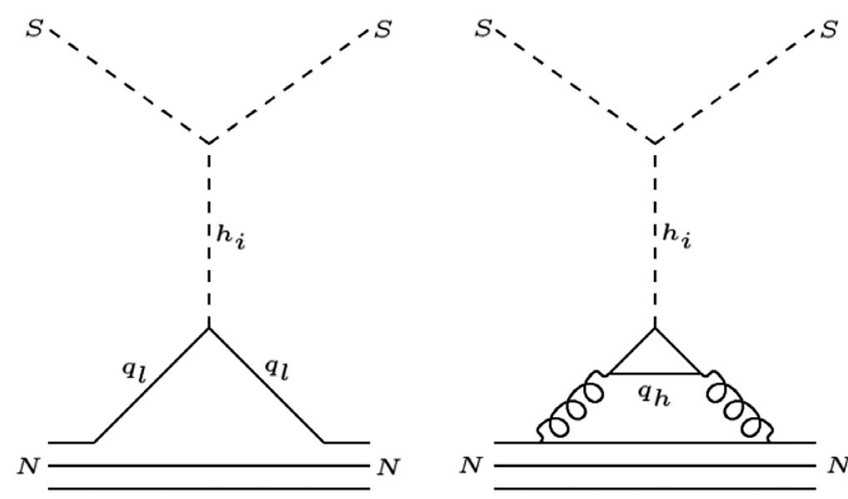

FIG. 1. The two leading order Feynman diagrams that contribute to the direct detection cross section. Left: tree-level scattering of the singlet $S$, through either the SM-like or heavy Higgs off of light quarks $q_{l}$. Right: scattering of the DM through loops of heavy quarks $q_{h}$ with the gluons in the nucleon.

nucleons, or through heavy quark loops with gluons, as shown in Fig. 1. The addition of a second Higgs doublet allows for a second mediator to this process and, generically, the scattering amplitudes can destructively interfere, leading to suppression in the direct detection bounds. This is one of the blind spots we consider below.

Low mass DM, $m_{S}<\frac{1}{2} m_{h}$, can also be produced at colliders through the decay of the SM-like Higgs $h \rightarrow S S$, which results in an invisible decay of the Higgs. Both the ATLAS and CMS experiments are searching for invisible Higgs decays and are setting bounds on the Higgs to the invisible branching ratio $[45,46]$. The most stringent direct bound comes from CMS and reads $\operatorname{BR}(h \rightarrow$ invisible $)$ $<19 \%$ [45]. The decay rate of $h \rightarrow S S$ is determined in large part by the coupling of $g_{S S h}$, the effective coupling of the Higgs to the DM. Similar to direct detection, there exist regions of parameter space in our model which make $g_{S S h}$ small, effectively avoiding invisible Higgs constraints. This is the second type of blind spot we will consider.

\section{A. Blind spots in direct detection}

First, we consider the blind spot in direct detection experiments. The spin-independent DM scattering cross section $\left(\sigma_{\mathrm{DM}}^{\mathrm{SI}}\right)$ on a nucleon $N$ reads

$$
\begin{aligned}
\sigma_{\mathrm{DM}}^{\mathrm{SI}} & =\frac{1}{8 \pi\left(m_{N}+m_{S}\right)^{2}} \\
& \times\left|\sum_{X=h, H} \frac{g_{S S X} m_{N}^{2}}{m_{X}^{2}}\left(\sum_{q=u, d, s} y_{q, X} f_{T q}+\sum_{q=c, b, t} \frac{2}{27} y_{q, X} f_{T G}\right)\right|^{2} .
\end{aligned}
$$

The parameters $y_{q, X}$ represent the couplings of the quarks to the SM-like Higgs and heavy Higgs and are given in Eqs. (3) and (4). The couplings of the DM to the Higgs bosons, $g_{S S X}$, are given in Eq. (7). The parameters $f_{T q}$ and
$f_{T G}$ represent the nucleon form factors for the quarks interacting with the nucleons in the detector [47], and other calculations of these parameters can be found in $[48,49]$. Blind spots occur for $\sigma_{\mathrm{DM}}^{\mathrm{SI}}=0$, so we must have that

$\frac{g_{S S h}}{g_{S S H}} \frac{m_{H}^{2}}{m_{h}^{2}}=-\frac{\sum_{q=u, d, s} y_{q, H} f_{T q}+\sum_{q=c, b, t} \frac{2}{27} y_{q, H} f_{T G}}{\sum_{q=u, d, s} y_{q, h} f_{T q}+\sum_{q=c, b, t} \frac{2}{27} y_{q, h} f_{T G}}$.

Note that to obtain this condition no statement has been made about the flavor structure of the 2HDM. Therefore, this cancellation is a generic feature of $2 \mathrm{HDM}+\mathrm{S}$ models and is ultimately fixed by the choice of quartic scalar couplings $\lambda_{S_{1}}, \lambda_{S_{2}}$, and $\lambda_{S_{12}}$; the flavor structure ("type") of 2HDM; and the 2HDM parameters $\alpha, \beta$, and $m_{H}$. Although this is a generic feature of any flavor structure, here we focus on the type $1 \mathrm{~A}$, type $1 \mathrm{~B}$, type $2 \mathrm{~A}$, and type $2 \mathrm{~B}$ structures. As discussed above, the type 1A/B and type 2A/B models represent the four ways of coupling the quarks in the standard flavor conserving $2 \mathrm{HDMs}$ and flavorful 2HDMs. By analyzing these four models we obtain a representative overview of the phenomenology of the blind spots in $2 \mathrm{HDM}+S$ models and how they are affected by the choice of flavor structure.

In Fig. 2 we show where the direct detection cancellation arises in the $\lambda_{S_{1}}$ vs $\lambda_{S_{2}}$ plane for an exemplary choice of the other model parameters: $m_{H}=300 \mathrm{GeV}, \cos (\beta-\alpha)=0$, $\tan \beta=5$, and $\lambda_{S_{12}}=0$. The choice $\cos (\beta-\alpha)=0$ [or more generally, $\cos (\beta-\alpha) \ll 1]$ corresponds to SM-like

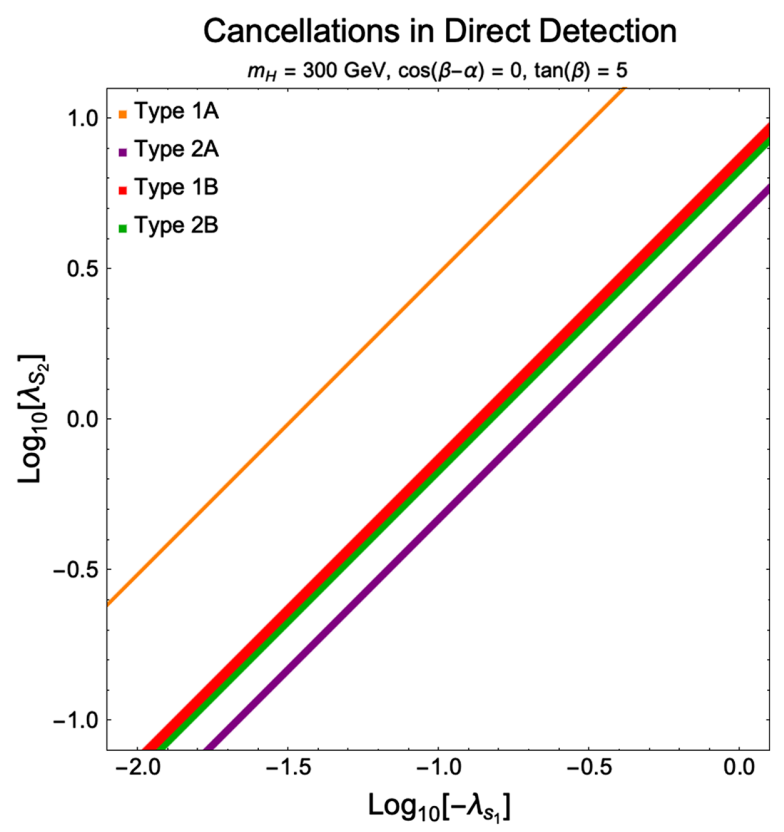

FIG. 2. Bands corresponding to the position of the direct detection blind spot in the plane of the quartic scalar couplings $\lambda_{S_{1}}$ and $\lambda_{S_{2}}$. The finite widths of the shown bands correspond to a variation of the nuclear form factors by $1 \sigma$. We show the blind spot regions in four types of 2HDMs: type 1A (orange line), 2A (purple line), 1B (red line), and 2B (green line). 
couplings of the light Higgs boson $h$. This is motivated by the good agreement of Higgs couplings measurements at the LHC with SM predictions [50-57]. Setting the coupling $\lambda_{S_{12}}$ to zero can be enforced by a Peccei-Quinn-type symmetry acting on the Higgs doublets [58].

The width of the bands in Fig. 2 corresponds to a $1 \sigma$ variation of the nucleon form factors [47]. For type 1A models the cancellation occurs for a larger hierarchy between $\lambda_{S_{1}}$ and $\lambda_{S_{2}}$ as compared to other types of models. This is because all the couplings to the heavy Higgs are subleading in this model, causing the cancellation between the diagrams occurring at smaller values of $g_{S S h}$, and hence generally smaller values of $\lambda_{S_{1}}$. The other three flavor structures all have similar values for quartic couplings in the cancellation regions as the heavy Higgs plays a larger role forcing $g_{S S h}$ to take on larger values than in the type 1A model. The precise location of the cancellation regions also depends on the choice of $\tan \beta$ and $m_{H}$. Larger values of $m_{H}$ generically require larger values of $\lambda_{S_{2}}$ for the cancellation to occur.

Note that the cancellation arises if one of the two quartic couplings $\lambda_{S_{1}}$ or $\lambda_{S_{2}}$ are negative. Negative terms in the potential can lead to the potential being unbounded from below, meaning there could exist field directions for which the potential goes to negative infinity. To study this possibility, we parametrize the three neutral scalar directions as follows:

$$
\begin{aligned}
S & =R \cos \theta, \\
\phi_{1}^{0} & =R \sin \theta \cos \phi, \\
\phi_{2}^{0} & =R \sin \theta \sin \phi,
\end{aligned}
$$

and study the positivity of the largest powers of $R$, which is $R^{4}$, in the potential on the sphere defined by the angles $\theta, \phi$. The requirement that the potential be positive as $R \rightarrow \infty$ then reads

$$
\begin{aligned}
\lambda_{S_{1}} & \sin \theta^{2} \cos \theta^{2} \cos \phi^{2}+\lambda_{S_{2}} \sin \theta^{2} \cos \theta^{2} \sin \phi^{2} \\
& +\lambda_{S} \cos \theta^{4}+2 \lambda_{S_{12}} \sin \theta^{2} \cos \theta^{2} \sin \phi \cos \phi \\
& +\frac{\lambda_{1}}{2} \sin \theta^{4} \cos \phi^{4}+\frac{\lambda_{2}}{2} \sin \theta^{4} \sin \phi^{4} \\
& +\lambda_{345} \sin \theta^{4} \cos \phi^{2} \sin \phi^{2}>0
\end{aligned}
$$

where the $\lambda_{i}, i=1, \ldots, 5$ are quartic couplings in the $2 \mathrm{HDM}$ potential as defined in [32] and $\lambda_{345}=$ $\lambda_{3}+\lambda_{4}+\lambda_{5}$. In the region of interest to us, $\lambda_{S_{1}}$ takes smaller values compared to $\lambda_{S_{2}}$. For this reason we take $\lambda_{S_{1}}$ to be negative; under this assumption and assuming that $\lambda_{S}$, $\lambda_{1}, \lambda_{2}, \lambda_{345}$ are $\mathcal{O}(1)$ and positive, then Eq. (13) can always be satisfied, and thus the potential is stable.

\section{B. Blind spots in invisible Higgs decays}

The second blind spot occurs for invisible Higgs decays. The decay width of the Higgs to the DM is given by

$$
\Gamma(h \rightarrow S S)=\frac{g_{S S h}^{2}}{32 \pi m_{h}}\left(1-4 \frac{m_{S}^{2}}{m_{h}^{2}}\right)^{1 / 2} .
$$

From this expression it is clear that we have a blind spot centered around $g_{S S h}=0$. Using Eq. (7) we see that this cancellation occurs when

$$
\frac{\lambda_{S_{1}}}{\lambda_{S_{2}}}=\frac{\tan \alpha}{\tan \beta},
$$

where we have set $\lambda_{S_{12}}=0$. For simplicity, we keep this choice for the remainder of the analysis, but note that this gives no fundamental difference to the analysis. Blind spots exist for any choice of $\lambda_{S_{12}}$ and are simply shifted in the parameter space when $\lambda_{S_{12}} \neq 0$.

By imposing that the invisible Higgs branching ratio $\mathrm{BR}(h \rightarrow S S)<0.19$ [45], we find that $g_{S S h} / v$ has to be less than $O(0.1)$ (the exact value changes depending on the choice of $m_{S}$ ). We show, in Fig. 3, under which conditions the direct detection cancellations overlap with parameter space where $g_{S S h}$ is sufficiently small to avoid invisible Higgs decay constraints. We show this for the DM matter mass of $m_{S}=45 \mathrm{GeV}$, with various choices for $\lambda_{S_{1}}$ and $\lambda_{S_{2}}$ (corresponding to the four panels in Fig. 3). Invisible Higgs decays exclude the region shaded in blue, with the exact cancellation line in dashed blue. The bound and the cancellation line depend on the choice of $\lambda_{S_{1}}$ and $\lambda_{S_{2}}$ but are independent of the type of 2HDM. Overlaid are the direct detection blind spots that occur for our four benchmark 2HDMs for two masses of the heavy Higgs $m_{H}=$ $300 \mathrm{GeV}$ or $m_{H}=1000 \mathrm{GeV}$. For a heavy Higgs mass of $300 \mathrm{GeV}$, the direct detection cancellation in the type $2 \mathrm{~A}$, type $1 \mathrm{~B}$, and type $2 \mathrm{~B}$ occurs for values of $\tan \beta$ outside the shown plot range.

The type 1A model avoids the constraints most easily as regardless of the parameters of the model the cancellation regions for direct detection and invisible Higgs decay are generally very similar. As mentioned above, in the type $1 \mathrm{~A}$ model the quarks primarily couple to the SM-Higgs and thus the direct detection cancellation is driven by $g_{S S h}$ being small just as invisible Higgs decay. For the other models we see that generally as $\lambda_{S_{1}}$ is lowered, the bound from the invisible Higgs decays is weakened. However, as we will see below, this also generally coincides with regions of parameter space where the DM is overabundant. With this in mind the most promising parameter space for "double blind spots" occurs for moderate values of $\lambda_{S_{1}}$ and $\lambda_{S_{2}}$. 

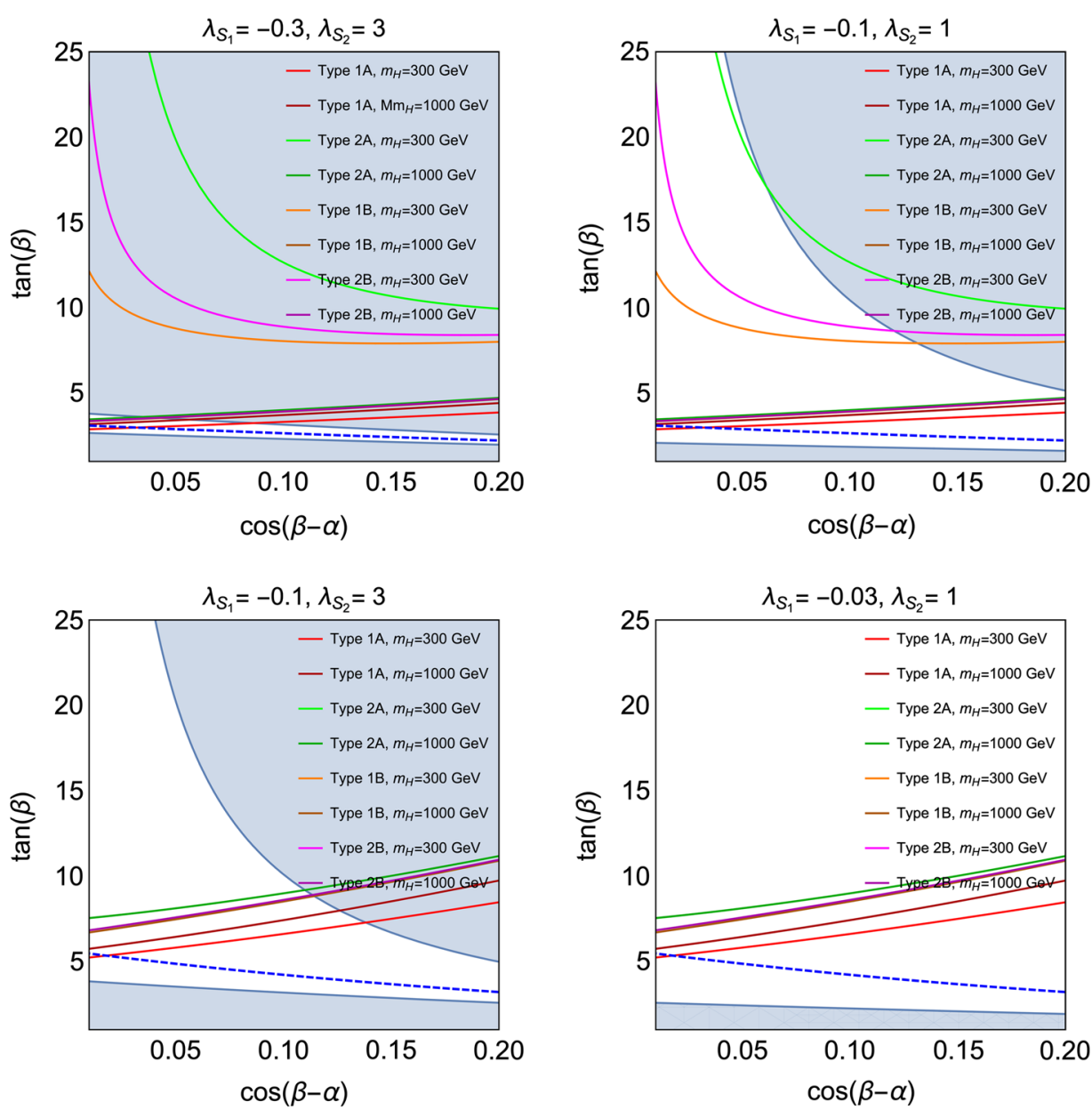

FIG. 3. Regions of parameter space in the $\cos \beta-\alpha$ vs $\tan \beta$ plane with blind spots for invisible Higgs decays for DM mass $m_{S}=45 \mathrm{GeV}$ and heavy Higgs mass $m_{H}=300 \mathrm{GeV}$ or $m_{H}=1000 \mathrm{GeV}$. The region excluded by invisible Higgs decays is shaded in blue, with the exact cancellation line in dashed blue. The bound and the cancellation line depend on the choice of $\lambda_{S_{1}}$ and $\lambda_{S_{2}}$ but are independent of the 2HDM flavor structure. Overlaid are the direct detection blind spots that occur for our four benchmark 2 HDMs.

\section{Fine-tuning of the blind spots}

The question of how "natural" the blind spots we point out are is connected to what extent the parameters must be finely tuned for those blind spots to occur. Fine-tuning refers to scenarios in which a single or several parameters must take on very specific values in order for a model to be consistent. The presence of accidental cancellations in our model could be associated with potentially large finetuning. One way to quantify the fine-tuning of a function is to employ the quantity [59]

$$
g(\vec{x})=\sum_{i=1}^{n}\left|\frac{x_{i}}{f\left(x_{i}\right)} \frac{\partial f\left(x_{i}\right)}{\partial x_{i}}\right|
$$

where $g(\vec{x})$ is the amount of tuning in the function $f(\vec{x})$. We show the fine-tuning of our model in Fig. 4, where $x_{i}=\lambda_{S_{i}}$, $i=1,2$, considering both the direct detection cross section and the invisible Higgs width. Generally, the tuning is mild in both models, but as expected the tuning gets very large directly at the cancellation lines. As we will discuss later based on current experimental constraints, one does not necessarily need to live exactly on this constraint, particularly for higher dark matter masses. So, there is still probable parameter space that does not suffer from large fine-tuning. However, for low mass DM the tuning can be quite large.

\section{PHENOMENOLOGY OF BLIND SPOTS}

In order to better understand the physical parameter space of the blind spots and the resulting phenomenology, we study the cancellations in the context of the four $2 \mathrm{HDM}$ discussed above (type $1 \mathrm{~A}$, type $2 \mathrm{~A}$, type $1 \mathrm{~B}$, type 2B). We implement the four models in the micrOMEGAs framework [60], modifying the default inert doublet model of micrOMEGAs to have the coupling structures under consideration, and use this to calculate the relic density and indirect detection limits. The direct detection cross section and invisible Higgs decay strengths are calculated analytically from the expressions in Eqs. (8) and (14). 

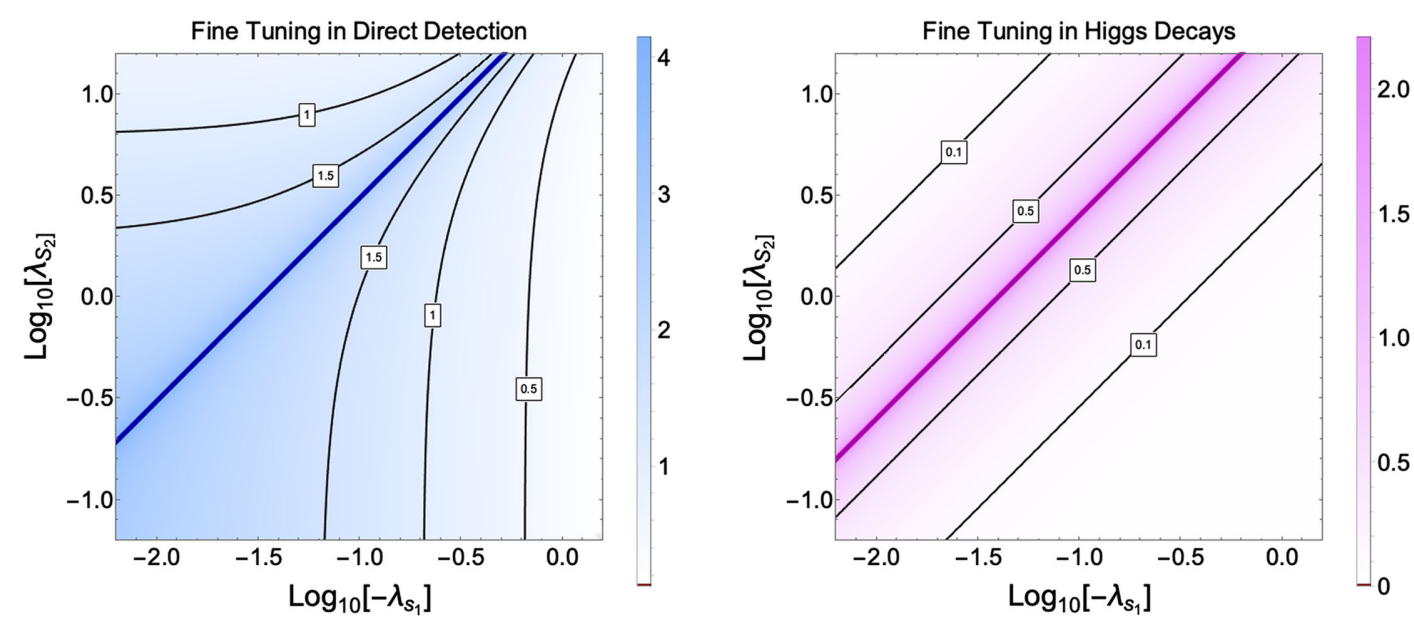

FIG. 4. The fine-tuning of the direct detection blind spot (left) and the invisible Higgs decay blind spot (right) in the $\lambda_{S_{1}}$ vs $\lambda_{S_{2}}$ plane, for the type 1A model. The darker regions represent areas of higher tuning. For direct detection the black lines show the contours for the tuning, and the blue line shows where the exact cancellation lies. For invisible Higgs decays the black lines show the contours for the tuning, and the pink line shows the exact cancellation.

We explore the parameter space of the quartic couplings $\lambda_{S_{1}}$ and $\lambda_{S_{2}}$ for various choices of the dark matter mass $m_{S}$, the $2 \mathrm{HDM}$ parameters $\cos (\beta-\alpha)$ and $\tan \beta$, and the type of $2 \mathrm{HDM}$.
In Fig. 5, we focus on the type $1 \mathrm{~A}$ model and vary $m_{S}=10,30,50,300,1000 \mathrm{GeV}$ for fixed $\cos (\beta-\alpha)=0$, $\tan \beta=5$ (as in Fig. 2). The white regions are allowed by all constraints. We see that generally as the DM mass is
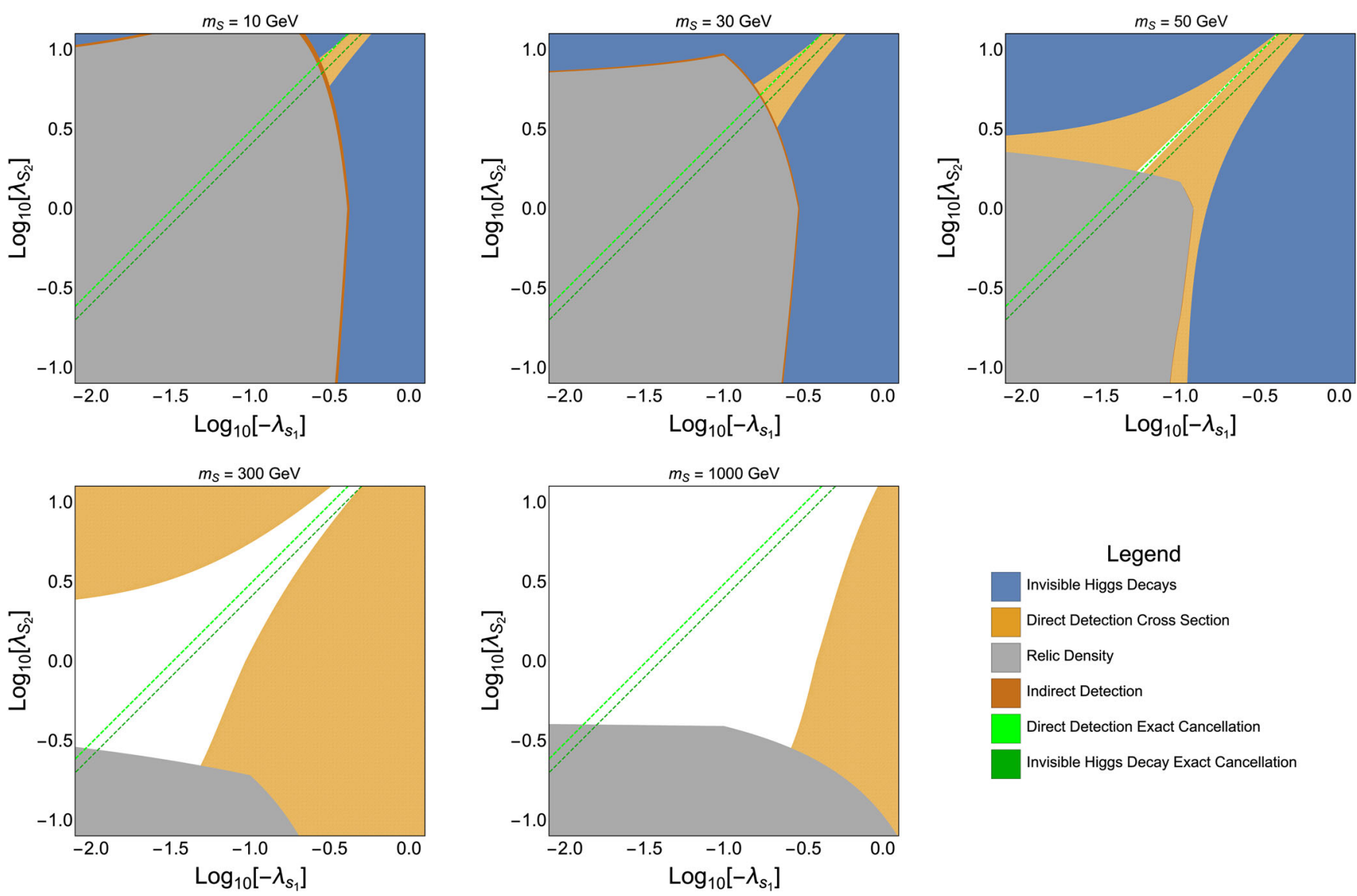

FIG. 5. Constraints in the $\lambda_{S_{1}}$ vs $\lambda_{S_{2}}$ plane in the type $1 \mathrm{~A}$ model for $\cos (\beta-\alpha)=0, \tan \beta=5, m_{H}=300 \mathrm{GeV}$, and various increasing values of dark matter mass $m_{S}$. The color coding of the various constraints is specified in the legend. 
increased, the constraints on the model are weakened. The two phenomenologically distinct regions of parameter space are when the DM mass is below and above half the Higgs mass. If $m_{S}>\frac{1}{2} m_{h}$, the constraints from invisible Higgs decays are automatically avoided. The constraints from direct detection are also particularly strong for the chosen lighter dark matter masses, $m_{S}=10,30,50 \mathrm{GeV}$. For those masses only a thin band close to the direct detection blind spot corresponds to viable parameter space. With this in mind, in the following we consider two benchmark masses of $m_{S}=45 \mathrm{GeV}$ and $m_{S}=300 \mathrm{GeV}$.

In Fig. 6 we show how the parameter space varies for different angles $\cos (\beta-\alpha)$ and $\tan \beta$. We observe that moderate values of $\tan \beta$ are favorable for these scenarios. As $\tan \beta$ gets small, the couplings of the quarks to the heavy Higgs increases (for the type 1A model), which causes destructive interference between the annihilation channels of DM through SM-like and heavy Higgs which constrains small values of $\tan \beta$ (this is specific to the type $1 \mathrm{~A}$ model). We do not find viable parameter space for $\tan \beta \gtrsim 10$ due to stronger constraints from direct detection and the relic density. This gives us a sweet spot for moderate values of $\tan \beta$ where the DM can efficiently annihilate in the early universe. $\cos (\beta-\alpha)$ has only a small impact on the results, making the relic density only slightly more constraining. Considering this along with the constraints on the 2HDM parameter space, as shown in [36], we focus on the benchmark case of $\cos (\beta-\alpha)=0, \tan \beta=5$ in the following.

In Fig. 7 we show the constraints for fixed dark matter mass $m_{S}=45$ in the different types of 2HDMs. In the type $2 \mathrm{~A}$, type $1 \mathrm{~B}$, and type $2 \mathrm{~B}$ models, the low $\mathrm{DM}$ mass regions are still highly constrained by the combination of relic density, direct detection, and invisible Higgs decays. In particular, in the region where DM is not overabundant the direct detection blind spots and the invisible Higgs blind spots do not overlap in these types. Only in the type $1 \mathrm{~A}$ model do we have a viable doubly blind spot, where direct detection and invisible Higgs decays are simultaneously avoided. However, in order for this doubly blind spot to occur we see that the fine-tuning must be quite high as shown in Fig. 4. Although the doubly blind spot only occurs for one of the benchmark models, it is still in stark contrast to simple SM $+S$ WIMP models where this region is ruled out by both direct detection and invisible Higgs decays.

Future experiments prove promising for the remaining parameter space of this low mass dark matter. Projections
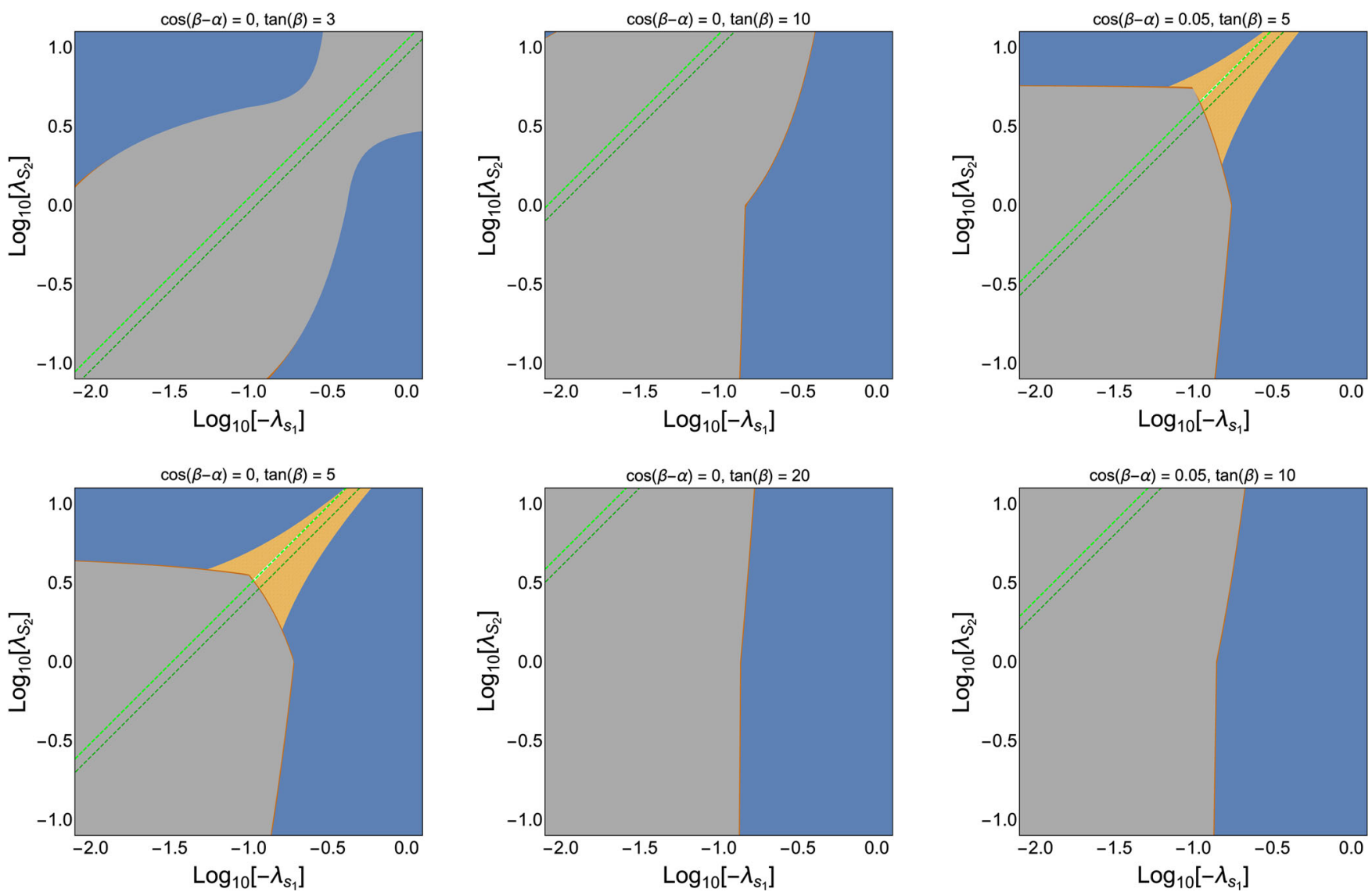

FIG. 6. Constraints in the $\lambda_{S_{1}}$ vs $\lambda_{S_{2}}$ plane in the type $1 \mathrm{~A}$ model for a dark matter mass $m_{S}=45 \mathrm{GeV}$ and heavy Higgs mass $m_{H}=300 \mathrm{GeV}$, varying the values for $\cos (\beta-\alpha), \tan \beta$. The color coding of the constraints is as in Fig. 5 . 

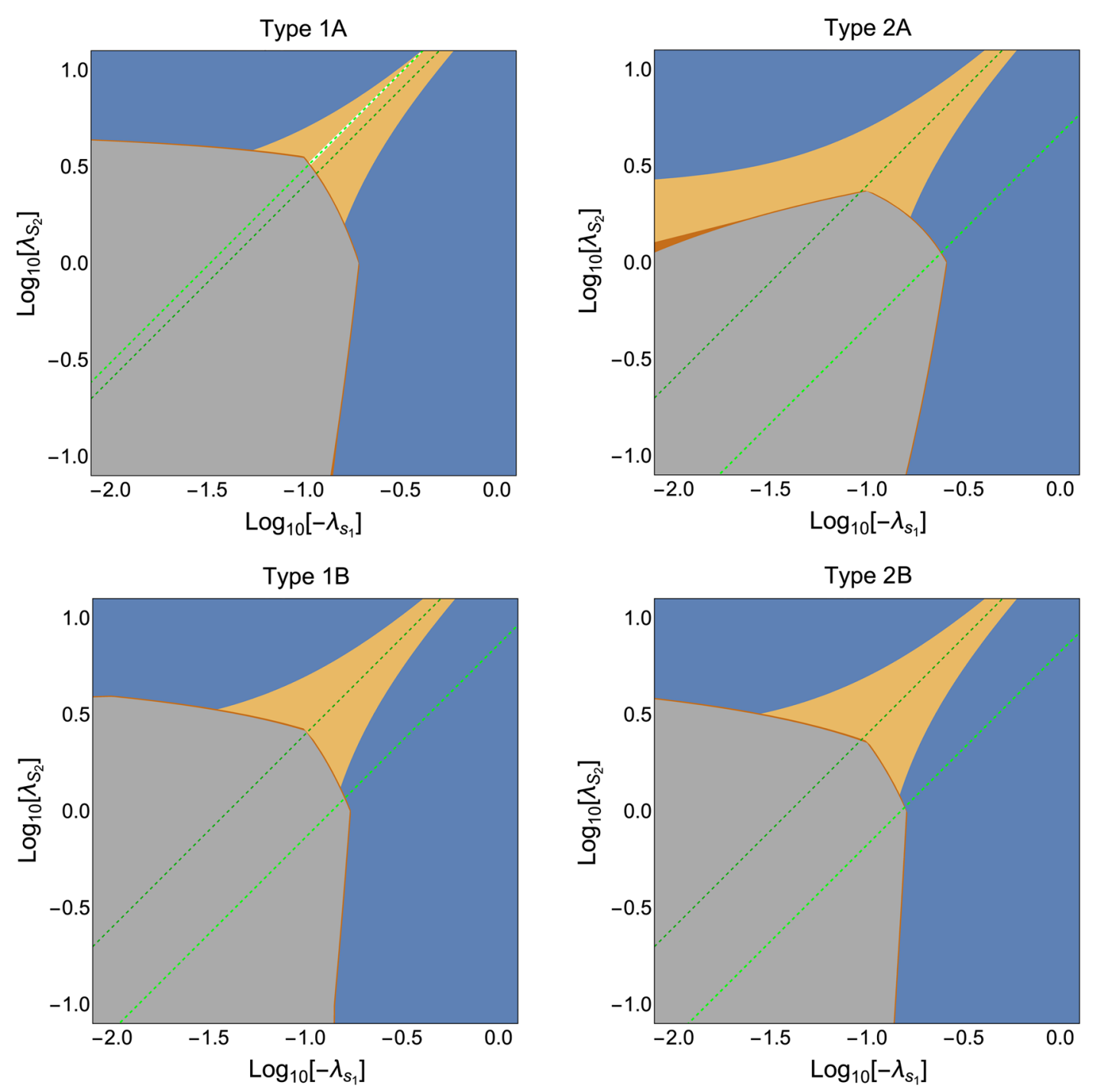

FIG. 7. Constraints in the $\lambda_{S_{1}}$ vs $\lambda_{S_{2}}$ plane for dark matter mass $m_{S}=45 \mathrm{GeV}$, heavy Higgs mass $m_{H}=300 \mathrm{GeV}$, $\cos (\beta-\alpha)=0$, and $\tan \beta=5$. We show type $1 \mathrm{~A}$ (top left), type $2 \mathrm{~A}$ (top right), type $1 \mathrm{~B}$ (bottom left), and type $2 \mathrm{~B}$ (bottom right). The color coding of the constraints is as in Fig. 5.

from the high luminosity LHC (HL-LHC) show a future sensitivity of $\mathrm{BR}(h \rightarrow S S)<0.025$ [61] and future direct detection experiments which will improve the measurement of the nucleon cross section by more than an order of magnitude $[62,63]$. Given this sensitivity nearly all masses such that $m_{S}<\frac{1}{2} m_{h}$ will be probed.

Finally the benchmark case of a heavy dark matter mass $m_{S}=300 \mathrm{GeV}$ is shown in Fig. 8 for the four types of 2HDMs. In this high mass region DM direct detection constraints are alleviated in a much larger portion of parameter space for all four flavor structures and the invisible Higgs constraint is completely absent. This shows that for a variety of flavor structure when the DM mass is high we can expect the direct detection blind spot to open up a large portion parameter space. This blind spot becomes more confined as one lowers the DM mass and more generous as one increases the mass. Similarly, the relic density constraints are much weaker for the high mass DM candidates. Overall, this leads to some viable parameter space in all four benchmark models. Additionally, the viable parameter space in the high DM mass regime can exist quite far from the cancellation lines, where the finetuning is low, unlike the low mass case where the viable parameter space only occurs in high fine-tuning regions. We also see that the nonstandard flavor structures of the type B models allow for an even more generous parameter space than the traditional flavor diagonal structures for the high mass DM benchmark. As discussed in the low mass case, future direct detections will continue to constrain this parameter space; however, without the additional constraint from invisible Higgs decays, there will still be a large amount of parameter space.

It is important to remember that for all DM masses explored above, the $\mathrm{SM}+S$ model is already excluded by 

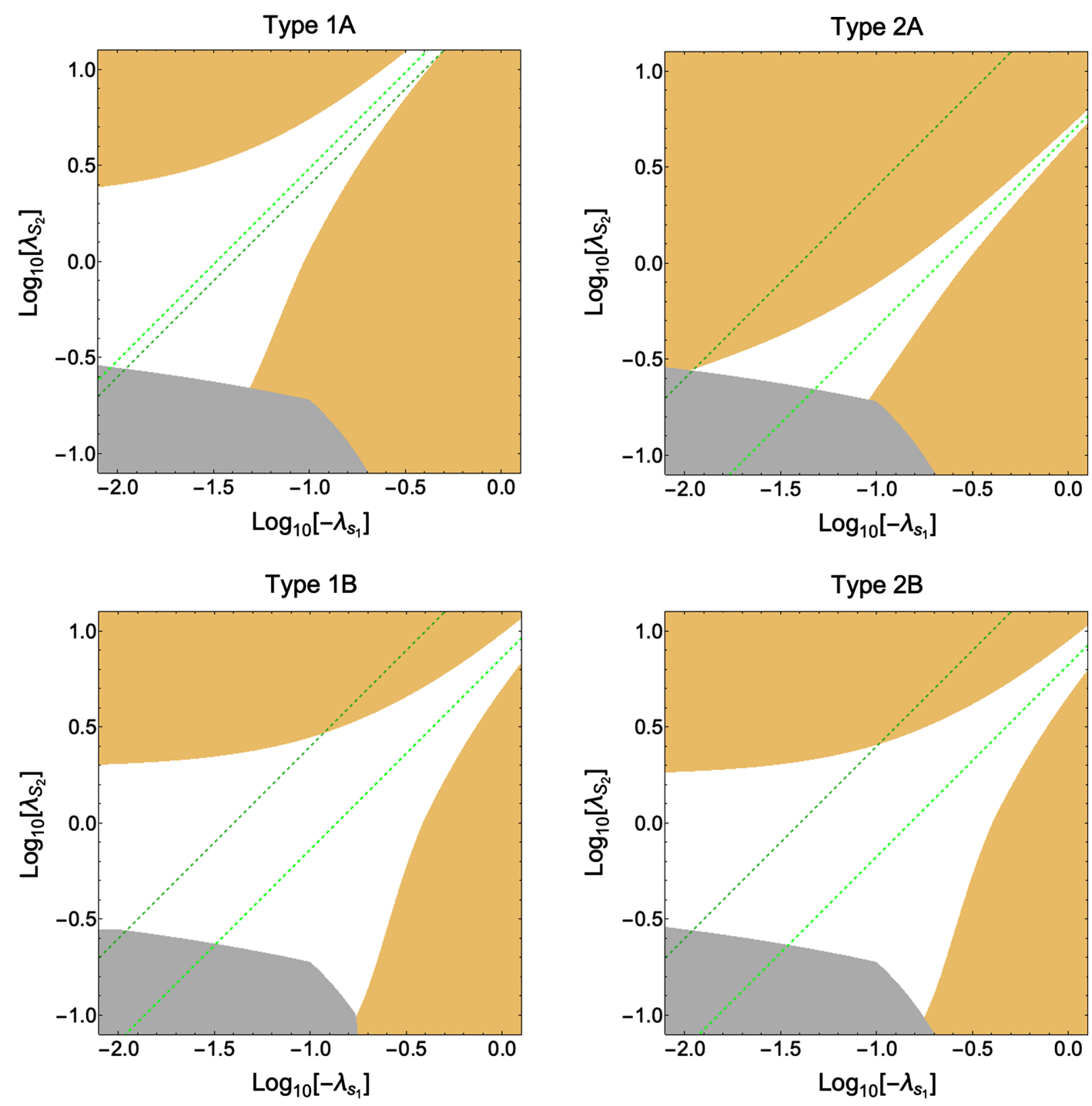

FIG. 8. Constraints in the $\lambda_{S_{1}}$ vs $\lambda_{S_{2}}$ plane for $m_{S}=300 \mathrm{GeV}, m_{H}=300 \mathrm{GeV}, \cos (\beta-\alpha)=0$, and tan $\beta=5$. We show type $1 \mathrm{~A}$ (top left), type 2A (top right), type $1 \mathrm{~B}$ (bottom left), and type $2 \mathrm{~B}$ (bottom right). The color coding of the constraints is as in Fig. 5.

either direct detection or invisible Higgs decays (outside of the resonant region). So, by adding a second Higgs doublet we provide regions of parameter space where DM candidates can exist at much lower masses than are possible in the $\mathrm{SM}+S$ case. Additionally, although we explored several fixed flavor structures, the cancellations can occur for any generic flavor structur, and are in no way associated with only the structures considered here.

\section{CONCLUSIONS}

The WIMP DM paradigm is a simple explanation to the question of the nature of the dark matter in the universe. However, in recent years such a paradigm has come under greater and greater pressure as a result of constraints from direct detection experiments as well as from results on the invisible Higgs decay modes. In this work we presented a model where one can take advantage of a second Higgs doublet in order to evade the constraints which invalidate most regions of parameter space of simpler WIMP models based on the existence of a singlet scalar field.

In particular, we find at DM masses below half the Higgs mass $m_{S}<\frac{1}{2} m_{h}$ that one can evade both direct detection and invisible Higgs decay constraints for flavor structures that are type 1A-like as a result of generic blind spots producing exact or approximate cancellations. Such cancellations depend in detail on the choice of the 2HDM parameters $\cos (\beta-\alpha), \tan \beta$, and $m_{H}$, but generally persist when the couplings of the fermions are primarily associated with the SM-like Higgs.

We also consider the scenario where the DM mass is large, $m_{S}>\frac{1}{2} m_{h}$, where we find that direct detection can be avoided for all considered 2HDM flavor structures. This primarily arises because one no longer needs to avoid the constraints imposed by invisible Higgs decays. Generically, we see that as the dark matter mass increases, the parameter 
space further opens up. There is also a weak dependence on the choice of $\cos (\beta-\alpha), \tan \beta$, and $m_{H}$. Mostly these choices affect the constraint of the relic density. Smaller values of $\cos (\beta-\alpha)$ and $\tan \beta$ typically result in a more open parameter space.

Overall, we find that with the inclusion of a second Higgs doublet one can access a much larger range of DM masses than in simpler models. This depends somewhat on the flavor structure of these models; however, in all the flavor structures considered, blind spots that facilitate the evasion of direct detection and collider constraints do exist, and, more generally, as we showed, blind spots can exist in any generic $2 \mathrm{HDM}$ setup.

\section{ACKNOWLEDGMENTS}

The research of W. A. and B. M. is supported by the National Science Foundation under Grant No. PHY1912719. S. P. is partly supported by the U.S. Department of Energy Grant No. de-sc0010107.
[1] J. Silk et al., Particle Dark Matter: Observations, Models and Searches (Cambridge University Press, Cambridge, England, 2010).

[2] S. Profumo, An Introduction to Particle Dark Matter (World Scientific, Singapore, 2017).

[3] J. de Swart, G. Bertone, and J. van Dongen, How dark matter came to matter, Nat. Astron. 1, 59 (2017).

[4] M. Cirelli, N. Fornengo, and A. Strumia, Minimal dark matter, Nucl. Phys. B753, 178 (2006).

[5] J. McDonald, Gauge singlet scalars as cold dark matter, Phys. Rev. D 50, 3637 (1994).

[6] S. Profumo, M. J. Ramsey-Musolf, and G. Shaughnessy, Singlet Higgs phenomenology and the electroweak phase transition, J. High Energy Phys. 08 (2007) 010.

[7] S. Profumo, L. Ubaldi, and C. Wainwright, Singlet scalar dark matter: Monochromatic gamma rays and metastable vacua, Phys. Rev. D 82, 123514 (2010).

[8] M. S. Boucenna and S. Profumo, Direct and indirect singlet scalar dark matter detection in the Lepton-Specific twoHiggs-doublet model, Phys. Rev. D 84, 055011 (2011).

[9] C. L. Wainwright, S. Profumo, and M. J. Ramsey-Musolf, Phase transitions and gauge artifacts in an Abelian Higgs plus singlet model, Phys. Rev. D 86, 083537 (2012).

[10] S. Profumo, M. J. Ramsey-Musolf, C. L. Wainwright, and P. Winslow, Singlet-catalyzed electroweak phase transitions and precision Higgs boson studies, Phys. Rev. D 91, 035018 (2015).

[11] L. Feng, S. Profumo, and L. Ubaldi, Closing in on singlet scalar dark matter: LUX, invisible Higgs decays and gamma-ray lines, J. High Energy Phys. 03 (2015) 045.

[12] W. Atwood et al., The large area telescope on the Fermi Gamma-ray space telescope mission, Astrophys. J. 697, 1071 (2009).

[13] J. Aleksic et al., Performance of the MAGIC stereo system obtained with Crab Nebula data, Astropart. Phys. 35, 435 (2012).

[14] J. Holder et al., Status of the VERITAS observatory, AIP Conf. Proc. 1085, 657 (2009).

[15] F. Aharonian et al., Observations of the Crab Nebula with H.E.S.S, Astron. Astrophys. 457, 899 (2006).

[16] D. S. Akerib et al., Results from a Search for Dark Matter in the Complete LUX Exposure, Phys. Rev. Lett. 118, 021303 (2017).
[17] G. Arcadi, M. Dutra, P. Ghosh, M. Lindner, Y. Mambrini, M. Pierre, S. Profumo, and F. S. Queiroz, The waning of the WIMP? A review of models, searches, and constraints, Eur. Phys. J. C 78, 203 (2018).

[18] C. Cheung, L. J. Hall, D. Pinner, and J. T. Ruderman, Prospects and blind spots for neutralino dark matter, J. High Energy Phys. 05 (2013) 100.

[19] P. Huang and C. E. M. Wagner, Blind spots for neutralino dark matter in the MSSM with an intermediate $m_{A}$, Phys. Rev. D 90, 015018 (2014).

[20] T. Han, F. Kling, S. Su, and Y. Wu, Unblinding the dark matter blind spots, J. High Energy Phys. 02 (2017) 057.

[21] T. Han, H. Liu, S. Mukhopadhyay, and X. Wang, Dark matter blind spots at one-loop, J. High Energy Phys. 03 (2019) 080.

[22] A. Crivellin, M. Hoferichter, M. Procura, and L. C. Tunstall, Light stops, blind spots, and isospin violation in the MSSM, J. High Energy Phys. 07 (2015) 129.

[23] M. Badziak, M. Olechowski, and P. Szczerbiak, Blind spots for neutralino dark matter in the NMSSM, J. High Energy Phys. 03 (2016) 179.

[24] X.-G. He, T. Li, X.-Q. Li, J. Tandean, and H.-C. Tsai, Constraints on scalar dark matter from direct experimental searches, Phys. Rev. D 79, 023521 (2009).

[25] X.-G. He, B. Ren, and J. Tandean, Hints of Standard Model Higgs Boson at the LHC and light dark matter searches, Phys. Rev. D 85, 093019 (2012).

[26] X.-G. He and J. Tandean, Low-mass dark-matter hint from CDMS II, Higgs Boson at the LHC, and Darkon models, Phys. Rev. D 88, 013020 (2013).

[27] X.-G. He and J. Tandean, New LUX and PandaX-II results illuminating the simplest Higgs-Portal dark matter models, J. High Energy Phys. 12 (2016) 074.

[28] C.-F. Chang, X.-G. He, and J. Tandean, Two-Higgs-doubletportal dark-matter models in light of direct search and LHC data, J. High Energy Phys. 04 (2017) 107.

[29] C.-F. Chang, X.-G. He, and J. Tandean, Exploring spin-3/2 dark matter with effective Higgs couplings, Phys. Rev. D 96, 075026 (2017).

[30] A. Greljo, J. Julio, J. F. Kamenik, C. Smith, and J. Zupan, Constraining Higgs mediated dark matter interactions, J. High Energy Phys. 11 (2013) 190. 
[31] G. Arcadi, 2HDM portal for singlet-doublet dark matter, Eur. Phys. J. C 78, 864 (2018).

[32] G. C. Branco, P. M. Ferreira, L. Lavoura, M. N. Rebelo, M. Sher, and J. P. Silva, Theory and phenomenology of twoHiggs-doublet models, Phys. Rep. 516, 1 (2012).

[33] S. L. Glashow and S. Weinberg, Natural conservation laws for neutral currents, Phys. Rev. D 15, 1958 (1977).

[34] W. Altmannshofer, S. Gori, A. L. Kagan, L. Silvestrini, and J. Zupan, Uncovering mass generation through Higgs flavor violation, Phys. Rev. D 93, 031301 (2016).

[35] W. Altmannshofer, J. Eby, S. Gori, M. Lotito, M. Martone, and D. Tuckler, Collider signatures of flavorful Higgs Bosons, Phys. Rev. D 94, 115032 (2016).

[36] W. Altmannshofer and B. Maddock, Flavorful two Higgs doublet models with a twist, Phys. Rev. D 98, 075005 (2018).

[37] W. Altmannshofer, B. Maddock, and D. Tuckler, Rare top decays as probes of flavorful Higgs Bosons, Phys. Rev. D 100, 015003 (2019).

[38] A. K. Das and C. Kao, A Two Higgs doublet model for the top quark, Phys. Lett. B 372, 106 (1996).

[39] A. E. Blechman, A. A. Petrov, and G. Yeghiyan, The flavor puzzle in multi-Higgs models, J. High Energy Phys. 11 (2010) 075.

[40] D. Ghosh, R. S. Gupta, and G. Perez, Is the Higgs mechanism of Fermion mass generation a fact? A Yukawa-less first-two-generation model, Phys. Lett. B 755, 504 (2016).

[41] F. J. Botella, G. C. Branco, M. N. Rebelo, and J. I. SilvaMarcos, What if the masses of the first two quark families are not generated by the standard model Higgs boson?, Phys. Rev. D 94, 115031 (2016).

[42] M. Blanke, S. Kast, J. M. Thompson, S. Westhoff, and J. Zurita, Spotting hidden sectors with Higgs binoculars, J. High Energy Phys. 04 (2019) 160.

[43] N. Aghanim et al., Planck 2015 results. XI. CMB power spectra, likelihoods, and robustness of parameters, Astron. Astrophys. 594, A11 (2016).

[44] T. Lin, TASI lectures on dark matter models and direct detection, Proc. Sci. TASI2018 (2019) 009 [arXiv: 1904.07915].

[45] A. M. Sirunyan et al., Search for invisible decays of a Higgs boson produced through vector boson fusion in protonproton collisions at $\sqrt{s}=13 \mathrm{TeV}$, Phys. Lett. B 793, 520 (2019).

[46] M. Aaboud et al., Combination of Searches for Invisible Higgs Boson Decays with the ATLAS Experiment, Phys. Rev. Lett. 122, 231801 (2019).

[47] G. Belanger, F. Boudjema, A. Pukhov, and A. Semenov, Dark matter direct detection rate in a generic model with micrOMEGAs 2.2, Comput. Phys. Commun. 180, 747 (2009).
[48] M. Hoferichter, J. R. de Elvira, B. Kubis, and U.-G. Meißner, High-Precision Determination of the Pion-Nucleon $\sigma$ Term from Roy-Steiner Equations, Phys. Rev. Lett. 115, 092301 (2015).

[49] M. Hoferichter, P. Klos, J. Menéndez, and A. Schwenk, Improved Limits for Higgs-Portal Dark Matter from LHC Searches, Phys. Rev. Lett. 119, 181803 (2017).

[50] G. Aad et al., Measurements of the Higgs boson production and decay rates and constraints on its couplings from a combined ATLAS and CMS analysis of the LHC pp collision data at $\sqrt{s}=7$ and $8 \mathrm{TeV}$, J. High Energy Phys. 08 (2016) 045.

[51] A. M. Sirunyan et al., Combined measurements of Higgs boson couplings in proton-proton collisions at $\sqrt{s}=$ 13 TeV, Eur. Phys. J. C 79, 421 (2019).

[52] M. Aaboud et al., Observation of Higgs boson production in association with a top quark pair at the LHC with the ATLAS detector, Phys. Lett. B 784, 173 (2018).

[53] A. M. Sirunyan et al., Observation of $\bar{t} \bar{t} H$ Production, Phys. Rev. Lett. 120, 231801 (2018).

[54] M. Aaboud et al., Observation of $H \rightarrow b \bar{b}$ decays and $V H$ production with the ATLAS detector, Phys. Lett. B 786, 59 (2018).

[55] A. M. Sirunyan et al., Observation of Higgs Boson Decay to Bottom Quarks, Phys. Rev. Lett. 121, 121801 (2018).

[56] A. M. Sirunyan et al., Observation of the Higgs boson decay to a pair of $\tau$ leptons with the CMS detector, Phys. Lett. B 779, 283 (2018).

[57] M. Aaboud et al., Cross-section measurements of the Higgs boson decaying into a pair of $\tau$-leptons in proton-proton collisions at $\sqrt{s}=13 \mathrm{TeV}$ with the ATLAS detector, Phys. Rev. D 99, 072001 (2019).

[58] R. D. Peccei and H. R. Quinn, Constraints imposed by CP conservation in the presence of instantons, Phys. Rev. D 16, 1791 (1977).

[59] R. Barbieri and G. F. Giudice, Upper bounds on supersymmetric particle masses, Nucl. Phys. B306, 63 (1988).

[60] G. Belanger, F. Boudjema, A. Pukhov, and A. Semenov, micrOMEGAs 3: A program for calculating dark matter observables, Comput. Phys. Commun. 185, 960 (2014).

[61] M. Cepeda et al., Higgs Physics at the HL-LHC and HE-LHC, arXiv:1902.00134.

[62] J. Liu, X. Chen, and X. Ji, Current status of direct dark matter detection experiments, Nat. Phys. 13, 212 (2017).

[63] S. Kang, S. Scopel, G. Tomar, and J.-H. Yoon, Present and projected sensitivities of dark matter direct detection experiments to effective WIMP-nucleus couplings, Astropart. Phys. 109, 50 (2019). 